\title{
Impact of the Baseline Anti-A/B Antibody Titer on the Clinical Outcome in ABO-Incompatible Kidney Transplantation
}

\author{
Byung Ha Chung ${ }^{a, b}$ Jeong Uk Lima,b Yaeni Kim ${ }^{a, b} \quad$ Ji-ll Kimª, c \\ In Sung Moon ${ }^{a, c}$ Bum Soon Choi ${ }^{a, b}$ Cheol Whee Park ${ }^{a, b}$ Yong-Soo Kim ${ }^{a, b}$ \\ Chul Woo Yang ${ }^{a, b}$ \\ ${ }^{a}$ Transplant Research Center, ${ }^{b}$ Division of Nephrology, Department of Internal Medicine, and ${ }^{c}$ Department of \\ Surgery, Seoul St. Mary's Hospital, College of Medicine, The Catholic University of Korea, Seoul, Korea
}

\section{Key Words}

ABO mismatch · Antibody titer $\cdot$ Kidney transplantation ·

Rituximab · Plasmapheresis

\begin{abstract}
Background/AIMS: We investigated the impact of the baseline anti-A/B antibody titer on the clinical outcome in ABOincompatible kidney transplantation (IKT). Methods: We included 183 patients who had undergone KT (40 ABO IKT and $143 \mathrm{ABO}$-compatible KT). Eight patients with a baseline titer of $\geq 1: 512$ were assigned to the high-titer group and $32 \mathrm{pa}$ tients with a baseline titer of $\leq 1: 256$ were assigned to the low-titer group. Patients who underwent ABO-compatible KT were used as the control group. We compared the clinical outcomes of the three groups. Results: Before transplantation, the high-titer group displayed more frequent antibody rebound, as shown in a lower titer reduction rate, and more difficulty reaching the target titer (1:16) than the low-titer group. During the postoperative period and out-clinic follow-up, antibody rebound was more frequent, and the rate of acute rejection and infection were significantly higher and allograft function was lower in the high-titer group than in the low-titer and control groups. Multivariate analysis
\end{abstract}

showed that high baseline antibody titer was an independent risk factor for acute rejection. Conclusion: ABO IKT in the high-titer group (baseline titer $\geq 1: 512$ ) required greater caution compared to the low-titer group because of the higher tendency of antibody rebound and the risk for acute rejection.

(c) 2013 S. Karger AG, Basel

\section{Introduction}

Recently, advances in immunosuppression and antibody removal protocols have made ABO-incompatible kidney transplantation (ABO IKT) feasible, and have increased the opportunities for patients to undergo transplantation [1-7]. Moreover, many recent studies have reported similar allograft and patient outcomes after ABOcompatible and incompatible kidney transplantation. Therefore, IKT has become a valuable option for patients with end-stage renal disease who do not have an $\mathrm{ABO}$ compatible donor $[1,3,4,6,8,9]$.

However, there are still several undetermined issues in ABO IKT. For example, the impact of the baseline anti-A/B antibody titer has not been established. In most

\section{KARGER}

E-Mail karger@karger.com

www.karger.com/nec
(C) 2013 S. Karger AG, Basel

$1660-2110 / 13 / 1242-0079 \$ 38.00 / 0$
Chul Woo Yang, MD

Department of Internal Medicine, Seoul St. Mary's Hospital

505 Banpo-Dong, Seocho-Ku

Seoul 137-040 (Korea)

E-Mail yangch@ catholic.ac.kr 
early reports of $\mathrm{ABO} \mathrm{IKT}$, the baseline anti-A/B antibody titer was an important predictor of acute antibodymediated rejection (AAMR) and allograft survival [1012]. A recent study also proposed that ABO IKT should not be recommended in patients with a baseline titer of $\geq 1: 512$ because of the risk of the failure for successful antibody removal [13]. However, another study has shown that a high baseline antibody titer is not predictive of a poor allograft outcome in patients treated with a tacrolimus-based triple immunosuppressant regimen [14].

In this study, we present our experiences of ABO IKT in patients with high baseline antibody titers compared their clinical outcomes with those of IKT patients with low baseline titer and control groups. We focus on the impact of the baseline anti-A/B antibody titer on the clinical outcomes of IKT patients.

\section{Patients and Methods}

\section{Study Population}

From April 2009 to February 2012, 197 living-donor KTs were performed in Seoul St. Mary's Hospital (Seoul, Korea). Of these, 14 patients who showed a positive result in any type of crossmatch test [complement-dependent cytotoxicity ( $\mathrm{T}$ and $\mathrm{B}$ cell) or flowcytometric crossmatch ( $\mathrm{T}$ and $\mathrm{B}$ cell)] or presence of donor-specific anti-human leukocyte antigen (HLA) antibody by Luminex single-antigen assay were excluded from this analysis. Thus, 183 patients who had undergone living-donor KT (40 ABO IKT and $143 \mathrm{ABO}$-compatible KT) were included in the study.

Of the 40 patients who underwent ABO IKT, 32 showed a baseline antibody titer equal to or less than 1:256, and were assigned to the low-titer group. Another 8 patients who showed a baseline titer equal to or higher than 1:512 were assigned to the high-titer group. The 143 patients who underwent ABO-compatible KT, with low immunological risk (no or low-grade donor-specific anti-HLA antibody), were used as the control group.

We retrospectively reviewed the medical records of those patients. We collected their baseline characteristics, including age, sex, the relationship between the recipient and donor, and the blood types of the donor and recipient. Peripheral-blood CD19 and CD20 cell counts were measured by flow cytometry before rituximab (RTX) (MabThera ${ }^{\mathrm{TM}}$; Genentech, Inc., San Francisco, Calif., USA) infusion and immediately before the initiation of plasmapheresis/intravenous immunoglobulin (PP/IVIG). We compared the changes in the anti-A/B antibody titer between high-titer and low-titer group during the pretransplantation plasmapheresis period. This study was approved by the Institutional Review Board of Seoul St. Mary's Hospital (KC11RCMI0716).

\section{Protocol for ABO IKT at Our Center}

Our center's protocol for ABO IKT has been described previously $[15,16]$. Briefly, we used RTX at a dose of 100 or 375 $\mathrm{mg} / \mathrm{m}^{2} 30$ days before transplantation. PP was performed using a COBE Spectra (Gambro BCT, Lakewood, Colo., USA) and the frequency of $\mathrm{PP}$ was planned based on the baseline titer, according to a previously recommended guideline [17]. At each PP session, one plasma volume was removed and replaced with a $5 \%$ albumin solution. From 1 day before transplantation to the posttransplantation period, we used a replacement fluid composed of $50 \%$ albumin (5\%) solution and $50 \%$ fresh-frozen plasma. IVIG (100-200 mg/kg) was infused $1 \mathrm{~h}$ after each PP session.

Anti-A/B antibody titers were determined using standard serological techniques, as described previously [15]. We measured both anti-A/B IgG and IgM, but we used IgG as the criteria to determine the pretransplant number of PP/IVIG and decide whether to reach target titer to allow KT. The anti-A/B antibody titer was measured every day from the start of PP/IVIG. The target titer at transplantation and during the first 2 weeks after transplantation was 1:16, but 1:32 was also acceptable if the titer did not reach $\leq 1: 16$ with the planned number of PP/IVIG sessions. When a patient underwent $\mathrm{KT}$ at a titer of 1:32, posttransplantation PP/IVIG was performed every other day until the antibody titer decreased to $\leq 1: 16$. When the titer rose above 1:16 within the first 2 weeks after KT, posttransplantation PP/IVIG was also performed until the antibody titer decreased to $\leq 1: 16$.

We began immunosuppression 7 days before transplantation. The immunosuppression regimen was composed of tacrolimus $(0.1 \mathrm{mg} / \mathrm{kg} /$ day $)$, mycophenolate mofetil (2 g/day), and prednisolone $(30 \mathrm{mg})$. The target trough level of tacrolimus was $8-12 \mathrm{ng} /$ $\mathrm{ml}$. Induction therapy using basiliximab $(20 \mathrm{mg})$ was administered on the day of surgery and on postoperative day 4 .

\section{Protocol Biopsy and Indication Biopsy}

An indication biopsy was performed in allograft patients with an increase in their sCr levels greater than $20 \%$. The biopsy procedure and histological diagnosis were as described previously [15].

\section{Comparison of the Clinical Outcome Parameters}

We compared the minimal number of PP/IVIG sessions required to reach the target titer (1:16) in the two groups. To compare the rebound in the antibody titer in each group, we calculated the titer reduction rate (TRR) using the following equation: $\mathrm{TRR}=$ (isoagglutinin titer step before initiation of PP - last titer step before transplantation)/number of PP/IVIG sessions. We also compared the changes in B cell counts and antibody titers before and after the administration of RTX. After transplantation, we compared the changes in the antibody titers of the two groups. We included the control group when comparing the changes in allograft function and the clinical outcomes, such as biopsy-proven acute rejection (acute $\mathrm{T}$ cell-mediated rejection and AAMR), infectious complications, and postoperative bleeding episodes.

\section{Statistical Analysis}

The statistical analyses were performed using SPSS software (version 15.0; IBM, Armonk, N.Y., USA). The data are presented as means \pm SD or counts and percentages, depending on the data type. For continuous variables, the means were compared using Student's t test. For categorical variables, Pearson's $\chi^{2}$ test and Fisher's exact test were used. All tests were two-tailed, and the results were considered significant at $\mathrm{p}<0.05$.
Chung/Lim/Kim/Kim/Moon/Choi/Park/ Kim/Yang 

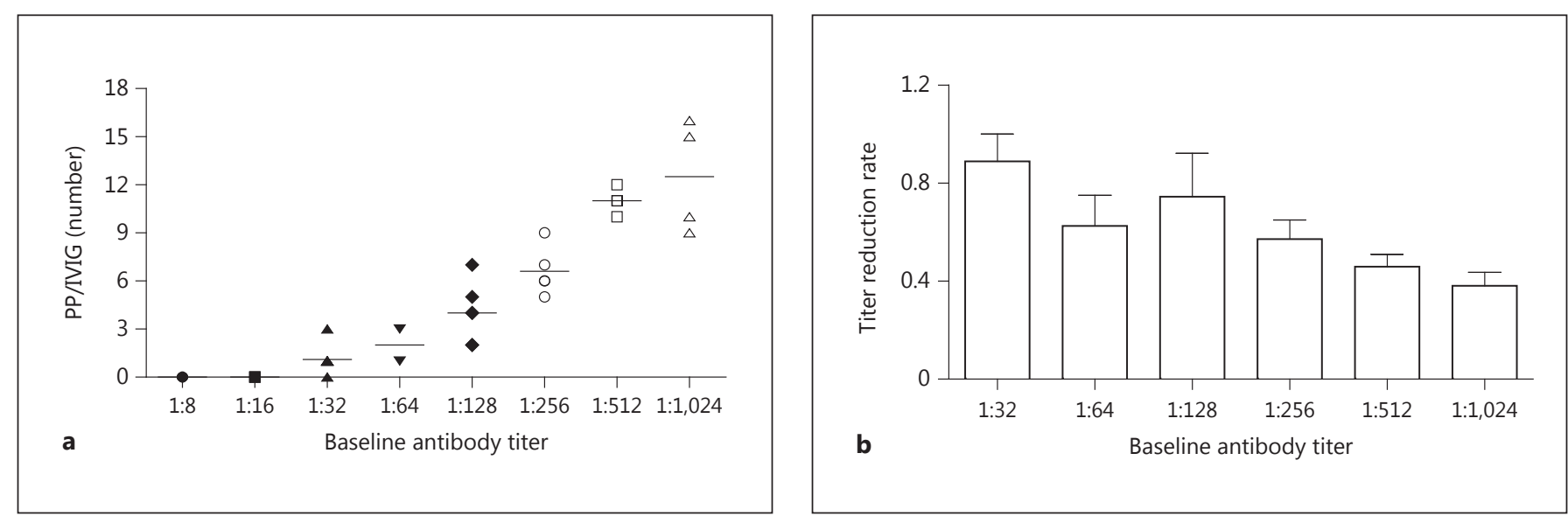

Fig. 1. a The minimal number of PP/IVIG sessions required to reach the target titer according to the baseline anti-A/B antibody titer. Note that the number of PP/IVIG sessions increased as the baseline titer increased. $\mathbf{b}$ The titer reduction rate according to the baseline antibody titer. The titer reduction rate decreased as the baseline antibody titer increased. c The anti-A/B antibody titer at transplantation according to the baseline titer. Most patients with a baseline antibody titer $\leq 1: 256$ underwent $\mathrm{KT}$ at an antibody titer of $<1: 16$. In contrast, most patients with a baseline titer $\geq 1: 512$ underwent $\mathrm{KT}$ at a titer of $1: 16$ or $1: 32$.

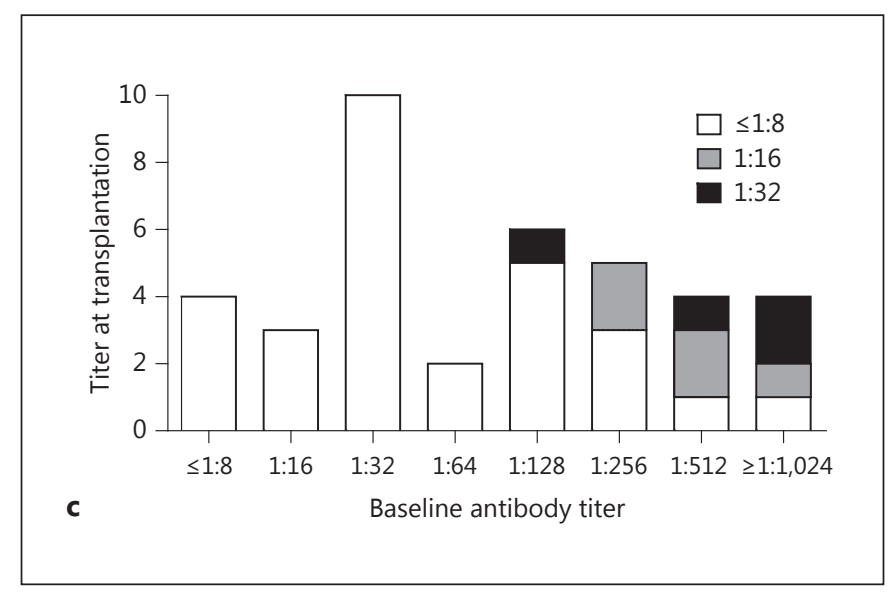

Table 1. Baseline characteristics of the patient populations

\begin{tabular}{|c|c|c|c|c|c|c|}
\hline & $\begin{array}{l}\text { Low titer } \\
(\mathrm{n}=32)\end{array}$ & $\begin{array}{l}\text { High titer } \\
(\mathrm{n}=8)\end{array}$ & $\begin{array}{l}\text { Control } \\
(n=143)\end{array}$ & $\begin{array}{l}\mathrm{p} \text { (low titer } \\
\text { vs. high titer) }\end{array}$ & $\begin{array}{l}\mathrm{p} \text { (low titer } \\
\text { vs. control) }\end{array}$ & $\begin{array}{l}\mathrm{p} \text { (high titer } \\
\text { vs. control) }\end{array}$ \\
\hline Age, years & $43.9 \pm 9.1$ & $46.9 \pm 6.7$ & $45.0 \pm 11.9$ & 0.27 & 0.68 & 0.51 \\
\hline F/U period, months & $18.8 \pm 9.8$ & $21.6 \pm 12.5$ & $21.9 \pm 10.0$ & 0.50 & 0.12 & 0.54 \\
\hline HLA mismatch & $3.5 \pm 1.3$ & $3.7 \pm 1.4$ & $2.9 \pm 1.7$ & 0.80 & 0.06 & 0.27 \\
\hline Donor, LRD/LURD & $19 / 13$ & $4 / 4$ & $114 / 46$ & 0.82 & 0.19 & 0.20 \\
\hline PRA $>50 \%$ & $10(31.2)$ & $1(12.5)$ & $21(14.7)$ & 0.41 & $<0.05$ & 0.67 \\
\hline \multicolumn{7}{|l|}{ Primary renal disease } \\
\hline Chronic GN & $8(25.0)$ & $1(12.5)$ & $54(33.8)$ & \multirow{4}{*}{0.13} & \multirow{4}{*}{0.41} & \multirow{4}{*}{0.17} \\
\hline $\mathrm{DM}$ & $9(28.1)$ & $2(25.0)$ & $34(21.2)$ & & & \\
\hline HTN & $9(28.1)$ & $3(37.5)$ & $25(15.6)$ & & & \\
\hline ADPKD & 0 & $1(12.5)$ & $4(2.5)$ & & & \\
\hline
\end{tabular}

F/U = Follow-up; LRD = living related donor; LURD = living unrelated donor; PRA = panel reactive antibody; GN = glomerulonephritis; DM = diabetes mellitus; HTN = hypertension; ADPKD = autosomal-dominant polycystic kidney disease. 
Fig. 2. Changes in CD19+/CD20+ cell counts and antibody titers before and after RTX infusion. In the low- (a, b) and hightiter groups (c, d), CD19+ and CD20+ cell counts were successfully depleted to less than $1 \%$. The antibody titer decreased after the infusion of RTX in both the low- (e) and the high-titer groups (f).

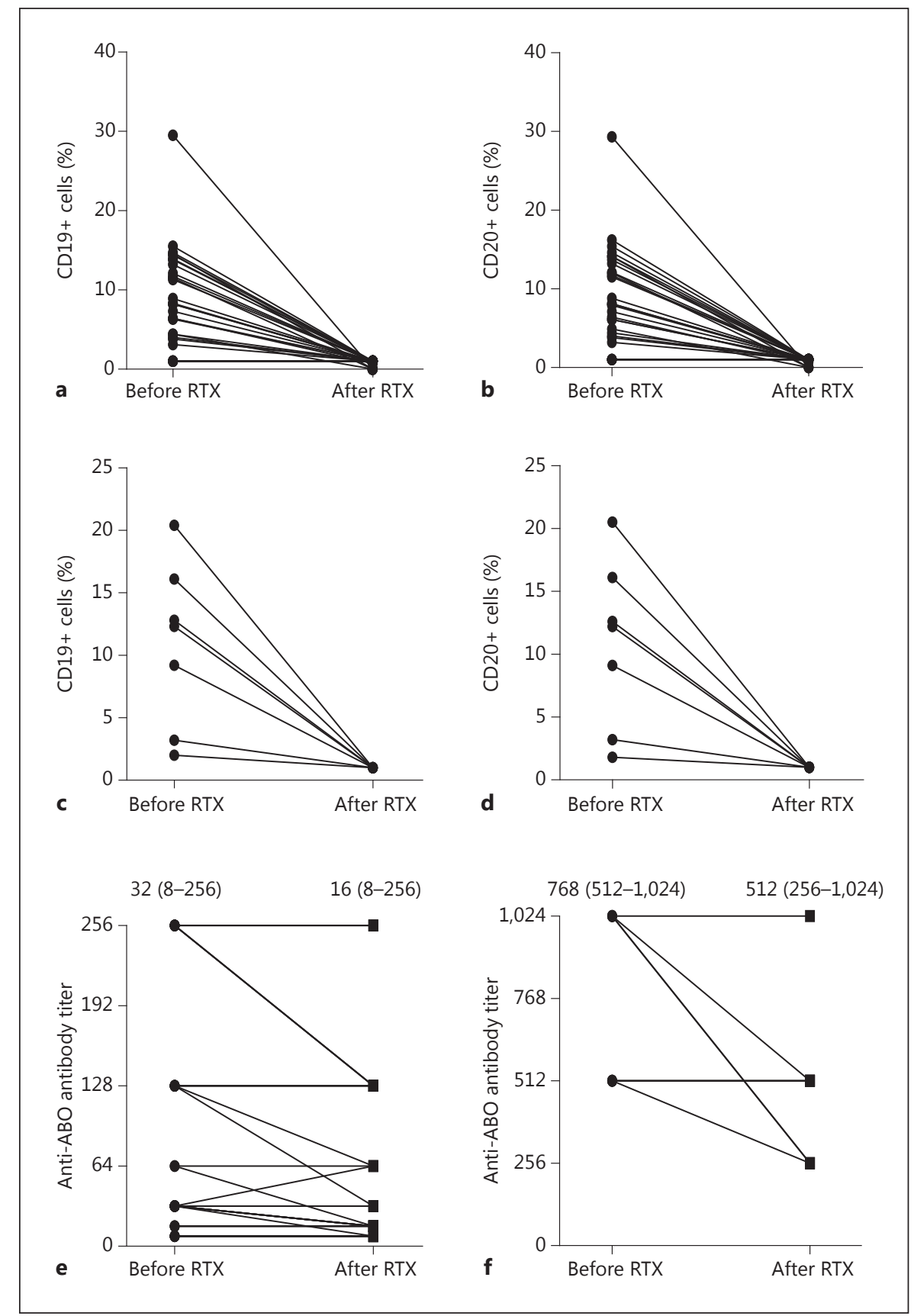

\section{Results}

\section{Clinical and Immunological Characteristics}

A comparison of the baseline characteristics of each group is shown in table 1. Age at transplantation, sex, duration of follow-up, donor type, incidence of retransplantation, and the distribution of primary renal disease did not differ in any pairwise comparison. The propor- tion of sensitized patients (panel reactive antibody $>50 \%$ ) was higher in the low-titer group than in the control group, but it did not differ in any other pairwise comparison (table 1). The median baseline anti-A/B antibody titer was significantly higher in the high-titer group $(1: 768[512-1024])$ than in the low-titer group $(1: 32[8-256] ; \mathrm{p}<0.05)$. 
Fig. 3. a Changes in the anti-A/B antibody (IgG) titer. A rebound in the antibody titer was detected during follow-up in the hightiter group at some time points. b Changes in serum creatinine levels in the patient groups. Serum creatinine was higher in the high-titer group at some time points than in the low-titer and control groups. ${ }^{*} \mathrm{p}<$ 0.05 versus low-titer group, ${ }^{* *} \mathrm{p}<0.05$ versus control group.

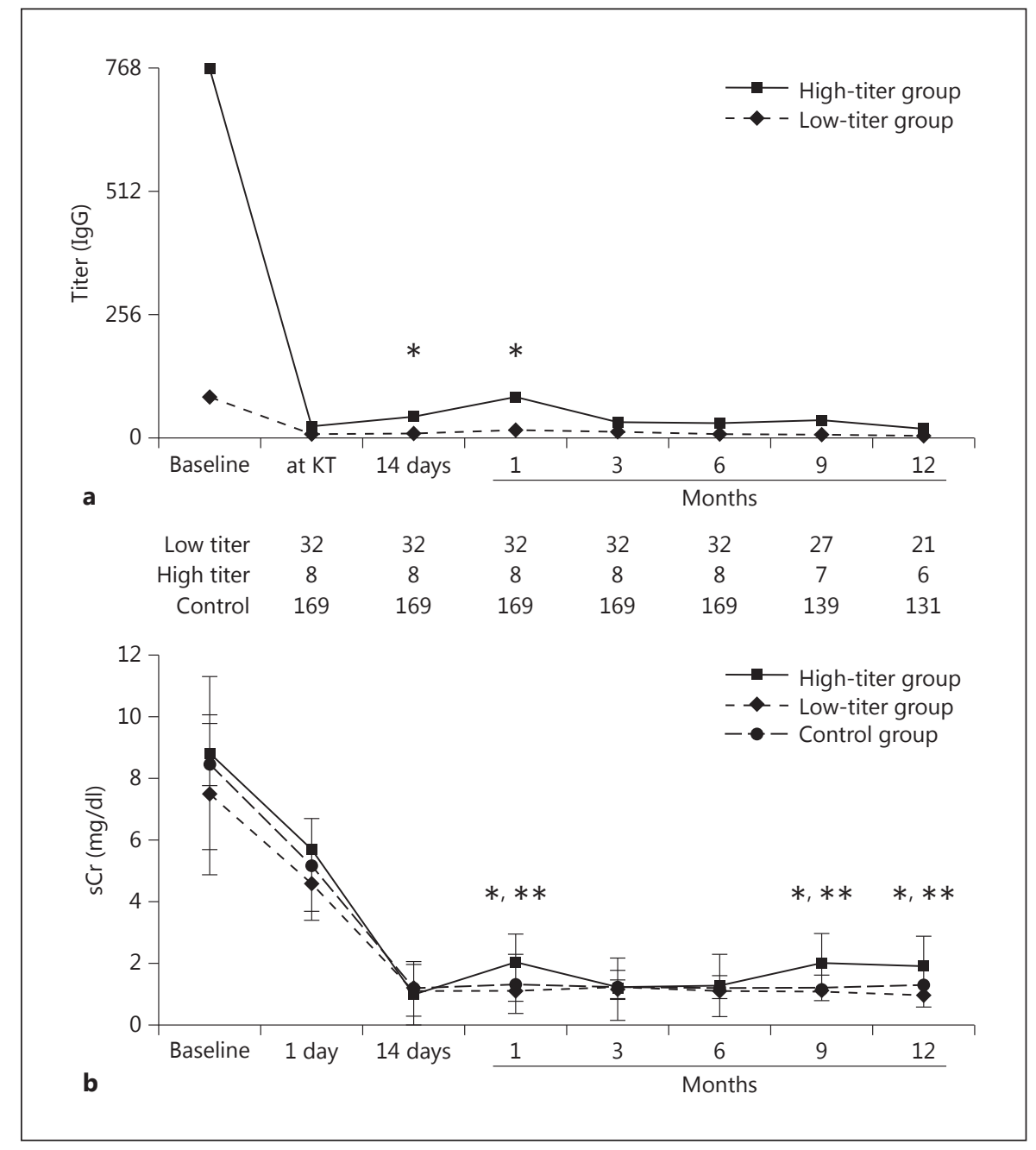

Numbers of PP/IVIG Sessions Required to Reach the

Target Titer and the Antibody Rebound Tendencies in the High- and Low-Titer Groups

We planned the number of PP/IVIG sessions according to a guideline recommended previously [17]. The minimal number of PP/IVIG sessions required to reach the target titer (1:16) increased exponentially according to the increase in the baseline antibody titer, and it was significantly higher in the high-titer group (11.8 \pm 2.5 sessions) than in the low-titer group ( $3.2 \pm 2.7$ sessions; $\mathrm{p}<$ 0.01 ; fig. 1a). TRR decreased as the baseline antibody titer increased, and was significantly lower in the high-titer group ( $0.44 \pm 0.16$ per session) than in the low-titer group $(0.75 \pm 0.33$ per session; $p=0.02$; fig. $1 b)$. In the low-titer group, the median antibody titer at transplantation was $1: 6(0-32)$ and the target titer $(\leq 1: 16)$ was reached with the planned number of PP/IVIG sessions in all but 1 pa- tient (3\%), who underwent KT with a titer of 1:32. The median antibody titer at transplantation in the high-titer group was 1:16 (2-32), which was higher than that in the low-titer group, and $37.5 \%$ (3/8) of patients underwent transplantation at a titer of $1: 32(\mathrm{p}<0.05$; fig. $1 \mathrm{c})$.

\section{Changes in Peripheral Blood B Cell Counts and} Antibody Titers after the Infusion of RTX in the High- and Low-Titer Groups

Before the infusion of RTX, the peripheral blood B cell (CD19+ and CD20+ cells) counts did not differ significantly between the low- and high-titer groups (CD19+ cells $9.2 \pm 5.0$ and $8.2 \pm 7.5 \%$, respectively, $\mathrm{p}=0.67$; CD20+ cells $9.3 \pm 5.2$ and $8.1 \pm 7.4 \%$, respectively, $\mathrm{p}=$ 0.62). After the infusion of RTX, the CD19+ and CD20+ cell counts were successfully depleted to less than $0.1 \%$ in both groups (fig. $2 \mathrm{a}-\mathrm{d}$ ). After the infusion of RTX, the 

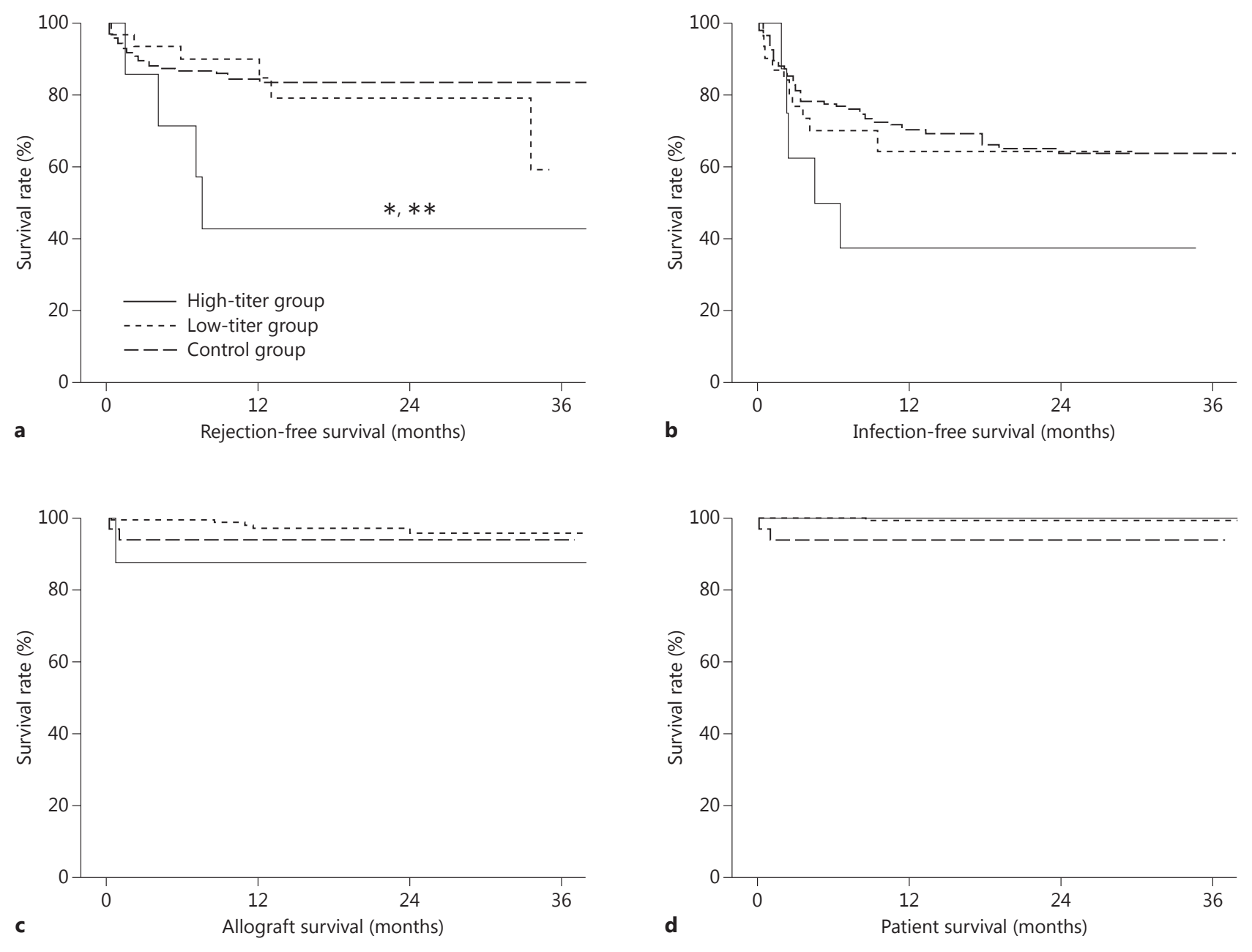

Fig. 4. Comparison of rejection-free survival rate (a), infectionfree survival rate (b), allograft survival rate (c), and patient survival rate (d). Note that no significant differences were detected in the allograft and patient survival rates of the three groups. However, the rejection-free survival rate was significantly lower in the

high-titer group than in the low-titer and control groups. The infection-free survival rate was lower in the high-titer group than in the other groups, although the difference was not statistically significant. ${ }^{*} \mathrm{p}<0.05$ versus low-titer group, ${ }^{* *} \mathrm{p}<0.05$ versus control group.

antibody titers decreased significantly in both the lowand high-titer groups ( $\mathrm{p}=0.04$, for both; fig. 3 ). In the high-titer group, it decreased from 1:768 $(512-1,024)$ to $1: 512(256-1,024)$ and in the low-titer group, it decreased from 1:32 (8-256) to 1:16 (8-256) (fig. 2e-f).

\section{Changes in Antibody Titers and Allograft Function after Transplantation}

Only 2 patients (5.0\%) in the low-titer group required posttransplantation PP/IVIG, whereas 3 of 8 patients (37.5\%) required posttransplantation PP/IVIG because

of antibody titer rebound over 1:16 during the first 2 weeks after surgery $(\mathrm{p}<0.05)$. In the low-titer group, no patient showed an antibody rebound over 1:32 during the outpatient clinic follow-up for 1 year after KT. In contrast, 2 of 8 patients (25\%) in the high-titer group showed an antibody titer rebound over 1:32, which resulted in AAMR $(p=0.09)$. Therefore, the median antibody titer was significantly higher in the high-titer group than in the low-titer group at some time points (fig. 3a). No difference in allograft function was detected between the lowtiter and control groups for 1 year after KT. However, a 
Table 2. Comparison of acute rejection and infectious complication

\begin{tabular}{|c|c|c|c|c|c|c|}
\hline Acute rejection & $5(15.6)$ & $5(62.5)$ & $23(16.1)^{*}$ & $<0.01$ & 0.59 & $<0.01$ \\
\hline ATCMR & $4(12.5)$ & $3(37.5)$ & $23(16.1)$ & 0.08 & 0.58 & 0.14 \\
\hline AAMR & $1(3.2)$ & $2(25.0)$ & $1(0.7)$ & 0.10 & 0.31 & $<0.01$ \\
\hline Infectious complication & $9(28.1)$ & $5(62.5)$ & $50(31.2)^{* *}$ & 0.06 & 1.0 & 0.09 \\
\hline Fungal infection & $1(3.2)$ & $1(12.5)$ & $0(0)$ & 0.37 & 0.17 & 0.05 \\
\hline Postoperative bleeding & $4(12.5)$ & $1(12.5)$ & $3(1.9)$ & 0.73 & 0.02 & 0.18 \\
\hline
\end{tabular}

Values are $\mathrm{n}(\%)$.

ATCMR = Acute T cell-mediated rejection.

* One patient suffered both ATCMR and AAMR; ** 11 patients suffered infection 2 times.

Table 3. Risk factors for the development of acute rejection

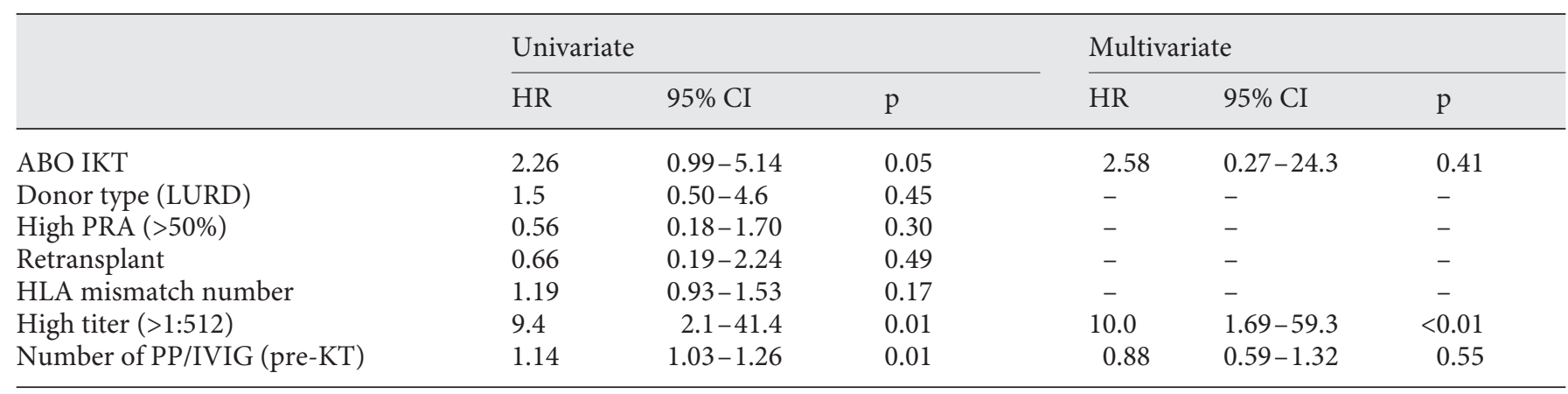

LURD = Living unrelated donor; PRA = panel reactive antibody.

significant increase in sCr was detected in the high-titer group compared with the low-titer and control groups at some time points during follow-up (fig. 3b).

\section{Development of Acute Rejections after KT}

The incidence of acute rejection was significantly higher in the high-titer group $(5 / 8,62.5 \%)$ than in the low-titer $(5 / 32,15.6 \%)$ or control groups $(23 / 143,16.8 \%$; $\mathrm{p}<0.05$, for all). The rejection-free survival rate was also significantly lower in the high-titer group than in the other groups ( $p<0.05$, for all; fig. $4 \mathrm{a}$ ). However, there were no differences between the low-titer and control groups. In the analysis of the acute rejection subtypes, the incidence of acute $\mathrm{T}$ cell-mediated rejection was higher in the high-titer than in the low-titer group, although the difference was not statistically significant $(p=0.09)$ The incidence of AAMR was significantly higher in the high-titer

High Baseline Titer in ABO-Incompatible Transplantation group than in the low-titer and control groups $(\mathrm{p}<0.05$, for all; table 2). Two cases of AAMR developed in the high-titer group. Allograft kidney biopsy showed typical peritubular capillaritis and diffuse C4d staining in the peritubular capillary which is compatible with the diagnosis of AAMR in both cases. We performed PP/IVIG in addition to steroid pulse therapy and the $\mathrm{sCr}$ level recovered to baseline value in both cases. In the multivariate analysis, a high baseline antibody titer $(\geq 1: 512)$ was also an independent risk factor for acute rejection (table 3 ).

\section{Development of Complications after KT}

The overall incidence of infectious complications was higher in the high-titer group than in the low-titer and control groups, and the infection-free survival rate was lower in the high-titer group, although the differences were not statistically significant ( $p=0.06$ vs. low-titer 
group, $\mathrm{p}=0.09$ vs. control; fig. $4 \mathrm{~b}$ ). The most common type of infection in the high-titer group was viral infection (2 CMV viremia and 2 herpes zoster infections). The incidence of postoperative bleeding was higher in both the low- $(\mathrm{p}<0.05)$ and high-titer groups $(\mathrm{p}=0.18)$ than in the control group. Four patients suffered postoperative bleeding in the low-titer group (12.5\%), but all cases were well controlled. In contrast, 1 patient in the high-titer group (12.5\%) suffered allograft nephrectomy because of uncontrolled bleeding (table 2).

\section{Patient and Allograft Outcomes after KT}

No significant differences were detected among the three groups in the allograft or patient survival rates (fig. 4c, d). There were 7 cases of allograft failure during follow-up: 2 in the low-titer group, 1 in the high-titer group, and 5 in the control group. The causes of both allograft failures in the low-titer group were patient death (sudden cardiac death and sepsis); the 1 failure in the high-titer group resulted from uncontrolled postoperative bleeding and there was no patient death in this group. In the control group, the failures were caused by 3 cases of acute rejection, 1 chronic rejection, and 1 patient death (trauma).

\section{Discussion}

In this study, we investigated the clinical impact of the baseline anti-A/B antibody titer to define the cutoff baseline titer for safe transplantation. Patients with a baseline titer of $\geq 1: 512$ before transplantation experienced antibody titer rebound significantly more frequently than patients with low baseline titers, which significantly hindered their reaching the target titer and entailed more pretransplantation PP/IVIG sessions. After transplantation, this rebound tendency still existed and was significantly associated with episodes of acute rejection.

Our first consideration was the definition of the 'high' baseline anti-A/B antibody titer. Some previous studies have defined a high titer as $\geq 1: 128$ or $\geq 1: 256$. However, these definitions were not based on previous clinical outcomes or systematic reviews, as the authors indicated [18, 19]. Therefore, those titers did not represent the cutoff values for ABO IKT or identify significant risk factors for adverse clinical outcomes after transplantation. In contrast, other groups have suggested that $\mathrm{ABO}$ IKT can be performed safely even in patients with extremely high baseline anti-A/B antibody titers [20,21]. However, these were reported as case studies and no study has been conducted on large numbers of patients. Therefore, those studies had many limitations, making it difficult to draw relevant conclusions from them.

The cut-off baseline titer for ABO IKT has been suggested in only one study [13]. The researchers proposed that $\mathrm{KT}$ was not recommended when the baseline titer was $\geq 1: 512$, because of the high rate of failure to reach the target anti-A/B antibody titer. However, they did not examine the clinical impact of the baseline titer after transplantation. In our previous pilot study, we could draw no clear conclusions about the role of high baseline titers because of the limited number of patients investigated, but we found an increasing tendency for acute rejection and other detrimental complications in patients with baseline titers of $\geq 1: 512$ [15]. Based on our previous experience and another group's report, we defined titers of $\geq 1: 512$ as 'high' titers in the context of ABO IKT in this study $[13,15]$.

Before transplantation, the first problem we encountered in ABO IKT with high baseline titers was the difficulty in reaching the target titer. As shown in figure 2a, the minimum number of PP/IVIG sessions required to reach the target titer increased exponentially with increases in the baseline titer. TRR also decreased significantly with increasing baseline antibody titers. In the lowtiter group, the target titer was achieved with fewer PP/ IVIG sessions than were initially planned according to a guideline reported previously [17]. In contrast, more PP/ IVIG sessions than were initially planned were required to reach the target titer in many patients in the high-titer group. As a consequence, a titer equal to or less than 1:16 was achieved in most patients in the low-titer group, but half the high-titer group underwent KT at a titer of 1:32 because prolonged PP/IVIG did not reduce the antibody titer to $1: 16$. This is consistent with a previous study that reported a high failure rate in reaching the target titer in patients whose baseline titer was $\geq 1: 512$ [13].

Interestingly, the response to RTX showed a similar pattern between the two groups. Peripheral blood B cells was significantly depleted with a single dose of RTX, and even more anti-A/B antibody titers showed a significant decrease after infusion of RTX compared to the baseline value. RTX can decrease a specific type of antibody in some previous reports through the mechanism that is not associated with the depletion of B cells [22]. In addition, RTX could deplete memory B cells, the precursors of plasma cells; hence, it may induce the decrease of short-lived plasma cells, which resulst in the decrease of anti-A/B antibody levels [23]. However, further investigation may be required to clarify this issue.

We investigated whether the rebound tendency in the antibody titer persisted after transplantation and whether
Chung/Lim/Kim/Kim/Moon/Choi/Park/ Kim/Yang 
it had any impact on acute rejection. During the postoperative period and the outpatient clinic follow-up, a significantly higher rate of antibody titer rebound was detected in the high-titer than in the low-titer group, in terms of the median titer and the proportion of patients who exceeded a titer of 1:32. It is noteworthy that 2 patients who showed an antibody titer rebound over 1:32 both suffered AAMR at the time of antibody rebound. Indeed, posttransplant rebound of anti-A/B antibody titer over 1:32 was significantly associated with AAMR in a previous study [24]. In addition, anti-HLA antibody was not detected by the Luminex single antigen assay, the most sensitive method for the detection of anti-HLA antibody $[25,26]$. Hence, we supposed that AAMR may develop due to rebound of the anti-A/B antibody titer. In contrast, 1 case of AAMR that developed in the low-titer group was associated with anti-HLA antibodies rather than anti-A/B antibodies, based on the high anti-HLA antibody level and the low anti-A/B antibody titer at the time of AMR.

Mostly because of the significant increase of AAMR, the overall prevalence of acute rejection was significantly higher in the high-titer group than in the low-titer and control groups. A high baseline antibody titer was also an independent risk factor for acute rejection. Consequently, allograft function deteriorated more strongly in the high-titer group than in the low-titer and control groups, perhaps attributable to the frequent development of acute rejection. Our results suggest that a high baseline anti-A/B antibody titer is associated with an increased risk of acute rejection because of the posttransplantation rebound in the antibody titer.

Intensive pretransplantation PP/IVIG is associated with various complications, including infection and postoperative bleeding $[27,28]$. Previous reports have demonstrated increased viral infection in ABO IKT with RTX and PP [27, 29-31]. In this study, the incidence of infection was higher in the high-titer group than in either the low-titer or control groups. It is possible that the more intensive PP/IVIG in the high-titer group resulted in a higher incidence of posttransplantation infection. In contrast, no difference was detected between the low-titer and control groups. Postoperative bleeding was more frequent in both the low- and high-titer groups than in the control group and uncontrolled bleeding requiring nephrectomy developed in the high-titer group. Our results suggest that strict monitoring of coagulation factors such fibrinogen, and early use of fresh-frozen plasma in plasma exchange may be required in ABO IKT.

This study does not insist that a high titer level is a contraindication for ABO IKT. Indeed, in spite of inferior clinical outcome in the high-titer group, the allograft and patient survival of this group did not differ from that of the low-titer and control groups. Hence, we suggest that differentiated protocol may be required in high-titer patients. First, an intensive immunosuppression regimen to suppress B cells may be necessary in the high-titer group, e.g. high-dose MMF may control humoral immunity and decrease antibody production $[35,36]$. Second, more intense anti-infection prophylaxis and careful monitoring for coagulation situation during the peritransplant period should be done. Third, immediate investigation for acute rejection when it was suspected and early treatment for it may prevent allograft loss due to acute rejection.

In conclusion, antibody titer rebound was frequent in the high-titer patients and was associated with a risk of failure to reach the target titer before transplantation and an increased risk of acute rejection and infection after transplantation compared to the low-titer group. Hence, $\mathrm{ABO}$ IKT in patients with a high baseline anti-A/B antibody titer $(\geq 1: 512)$ required greater caution compared to the low-titer group to prevent various complications and improve the clinical outcomes of this group.

\section{Acknowledgment}

This study was supported by a grant (A092258) from the Korea Healthcare Technology R\&D Project, Ministry for Health, Welfare \& Family Affairs, Republic of Korea.

\section{Disclosure Statement}

No conflict of interest.

References

Nephron Clin Pract 2013;124:79-88 DOI: $10.1159 / 000355855$
1 Ichimaru N, Takahara S: Japan's experience with living-donor kidney transplantation across ABO barriers. Nat Clin Pract Nephrol 2008;4:682-692.

-2 Gloor JM, Stegall MD: Abo incompatible kidney transplantation. Curr Opin Nephrol Hypertens 2007; 16:529-534.

-3 Sonnenday CJ, Warren DS, Cooper M, Samaniego M, Haas M, King KE, Shirey RS, Simpkins CE, Montgomery RA: Plasmapheresis, CMV hyperimmune globulin, and antiCD20 allow ABO-incompatible renal transplantation without splenectomy. Am J Transplant 2004;4:1315-1322.

$\checkmark 4$ Genberg H, Kumlien G, Wennberg L, Berg U, Tydn G: ABO-incompatible kidney transplantation using antigen-specific immunoadsorption and rituximab: a 3-year follow-up. Transplantation 2008;85:1745-1754. 
-5 Tyden G, Donauer J, Wadstrom J, Kumlien G, Wilpert J, Nilsson T, Genberg H, Pisarski P, Tufveson G: Implementation of a protocol for $\mathrm{ABO}$-incompatible kidney transplantation a three-center experience with 60 consecutive transplantations. Transplantation 2007;83: 1153-1155.

-6 Tyden G, Kumlien G, Genberg H, Sandberg J, Lundgren T, Fehrman I: Abo incompatible kidney transplantations without splenectomy, using antigen-specific immunoadsorption and rituximab. Am J Transplant 2005;5: 145-148.

7 Ishida H, Miyamoto N, Shirakawa H, Shimizu $\mathrm{T}$, Tokumoto T, Ishikawa N, Shimmura $\mathrm{H}$, Setoguchi K, Toki D, Iida S, Teraoka S, Takahashi K, Toma H, Yamaguchi Y, Tanabe K: Evaluation of immunosuppressive regimens in $\mathrm{ABO}$-incompatible living kidney transplantation - single center analysis. Am J Transplant 2007;7:825-831.

${ }_{8}$ Tanabe $\mathrm{K}$, Tokumoto $\mathrm{T}$, Ishida $\mathrm{H}$, Ishikawa $\mathrm{N}$, Miyamoto N, Kondo T, Shimmura H, Setoguchi K, Toma H: Excellent outcome of $\mathrm{ABO}$-incompatible living kidney transplantation under pretransplantation immunosuppression with tacrolimus, mycophenolate mofetil, and steroid. Transplant Proc 2004;36: 2175-2177.

-9 Takahashi K, Saito K, Takahara S, Okuyama A, Tanabe K, Toma H, Uchida K, Hasegawa A, Yoshimura N, Kamiryo Y: Excellent longterm outcome of $\mathrm{ABO}$-incompatible living donor kidney transplantation in Japan. Am J Transplant 2004;4:1089-1096.

$\checkmark 10$ Gloor JM, Stegall MD: ABO incompatible kidney transplantation. Curr Opin Nephrol Hypertens 2007;16:529-534.

-11 Stegall MD, Dean PG, Gloor JM: ABO-incompatible kidney transplantation. Transplantation 2004;78:635-640.

12 Gloor JM, Lager DJ, Moore SB, Pineda AA, Fidler ME, Larson TS, Grande JP, Schwab TR, Griffin MD, Prieto M, Nyberg SL, Velosa JA, Textor SC, Platt JL, Stegall MD: ABO-incompatible kidney transplantation using both $\mathrm{A} 2$ and non-A2 living donors. Transplantation 2003;75:971-977.

13 Lawrence C, Galliford JW, Willicombe MK, McLean AG, Lesabe M, Rowan F, Papalois V, Regan F, Taube D: Antibody removal before $\mathrm{ABO}$-incompatible renal transplantation: how much plasma exchange is therapeutic? Transplantation 2011;92:1129-1133.

14 Shimmura H, Tanabe K, Ishida H, Tokumoto T, Ishikawa N, Miyamoto N, Shirakawa H, Setoguchi K, Nakajima I, Fuchinoue S, Teraoka S, Toma H: Lack of correlation between results of $\mathrm{ABO}$-incompatible living kidney transplantation and anti-ABO blood type antibody titers under our current immunosuppression. Transplantation 2005;80:985988.
5 Chung BH, Lee JY, Kang SH, Sun IO, Choi SR, Park HS, Kim JI, Moon IS, Choi BS, Park CW, Kim YS, Yang CW: Comparison of clinical outcome between high and low baseline anti-ABO antibody titers in ABO-incompatible kidney transplantation. Ren Fail 2011;33:150-158.

$\checkmark 16$ Chung BH, Hong YA, Sun IO, Piao SG, Kim JI, Moon IS, Choi BS, Park CW, Kim YS, Yang $\mathrm{CW}$ : Determination of rituximab dose according to immunologic risk in $\mathrm{ABO}$-incompatible kidney transplantation. Ren Fail 2012; 34:974-979.

17 Tobian AA, Shirey RS, Montgomery RA, Tisch DJ, Ness PM, King KE: Therapeutic plasma exchange reduces abo titers to permit ABO-incompatible renal transplantation. Transfusion 2009;49:1248-1254.

18 Montgomery RA: Renal transplantation across HLA and ABO antibody barriers: integrating paired donation into desensitization protocols. Am J Transplant 2010;10:449-457.

19 Wilpert J, Geyer M, Teschner S, Schaefer T, Pisarski P, Schulz-Huotari C, Gropp A, Wisniewski U, Goebel H, Gerke P, Walz G, Donauer J: ABO-incompatible kidney transplantation-proposal of an intensified apheresis strategy for patients with high initial isoagglutinin titers. J Clin Apher 2007;22:314-322.

20 Koshino K, Okamoto M, Sakai K, Suzuki T, Nobori S, Matsuyama M, Ushigome H, Okajima $\mathrm{H}$, Yoshimura $\mathrm{N}$ : The excellent outcomes of ABO-incompatible kidney transplantation with high titer $(>\times 2048)$ using anti-CD20 and anti-CD25 antibody without splenectomy: two case reports. Transplant Proc 2011;43:2379-2382.

21 Uchida J, Iwai T, Kato M, Machida Y, Naganuma T, Kumada N, Yoshimura R, Kawashima H, Kim T, Nakatani T: A novel approach to successful ABO-incompatible hightiter renal transplantation. Transplant Proc 2008;40:2285-2288.

22 Pescovitz MD: Rituximab, an anti-CD20 monoclonal antibody: history and mechanism of action. Am J Transplant 2006;6:859866.

23 Shapiro-Shelef M, Calame K: Regulation of plasma-cell development. Nat Rev Immunol 2005;5:230-242.

24 Tobian AA, Shirey RS, Montgomery RA, Cai W, Haas M, Ness PM, King KE: Abo antibody titer and risk of antibody-mediated rejection in $\mathrm{ABO}$-incompatible renal transplantation. Am J Transplant 2010;10:1247-1253.

25 Eng HS, Bennett G, Tsiopelas E, Lake M, Humphreys I, Chang SH, Coates PT, Russ GR: Anti-HLA donor-specific antibodies detected in positive B-cell crossmatches by Luminex predict late graft loss. Am J Transplant 2008;8:2335-2342.

26 Vlad G, Ho EK, Vasilescu ER, Colovai AI, Stokes MB, Markowitz GS, D'Agati VD, Cohen DJ, Ratner LE, Suciu-Foca N: Relevance of different antibody detection methods for the prediction of antibody-mediated rejection and deceased-donor kidney allograft survival. Hum Immunol 2009;70:589-594.
Crew RJ, Ratner LE: Abo-incompatible kidney transplantation: current practice and the decade ahead. Curr Opin Organ Transplant 2010;15:526-530.

28 Shin M, Kim SJ: Abo incompatible kidney transplantation: current status and uncertainties. J Transplant 2011;2011:970421.

29 Grim SA, Pham T, Thielke J, Sankary H, Oberholzer J, Benedetti E, Clark NM: Infectious complications associated with the use of rituximab for $\mathrm{ABO}$-incompatible and positive cross-match renal transplant recipients. Clin Transplant 2007;21:628-632.

30 Aikawa A, Ohara T, Arai K, Hadano T, Kawamura T, Sugiyama K, Muramatsu M, Itabashi Y, Kawada N, Kanai T, Hasegawa A: Clinical outcome and accommodation in abo incompatible kidney transplantation. Clin Transplant 2004:135-142.

31 Habicht A, Broker V, Blume C, Lorenzen J, Schiffer M, Richter N, Klempnauer J, Haller $\mathrm{H}$, Lehner F, Schwarz A: Increase of infectious complications in $\mathrm{ABO}$-incompatible kidney transplant recipients - a single centre experience. Nephrol Dial Transplant 2011;26:41244131.

32 Solez K, Colvin RB, Racusen LC, Haas M, Sis B, Mengel M, Halloran PF, Baldwin W, Banfi G, Collins AB, Cosio F, David DS, Drachenberg C, Einecke G, Fogo AB, Gibson IW, Glotz D, Iskandar SS, Kraus E, Lerut E, Mannon RB, Mihatsch M, Nankivell BJ, Nickeleit V, Papadimitriou JC, Randhawa P, Regele H, Renaudin K, Roberts I, Seron D, Smith RN, Valente M: Banff 07 classification of renal allograft pathology: updates and future directions. Am J Transplant 2008;8:753-760.

33 Setoguchi K, Ishida H, Shimmura H, Shimizu T, Shirakawa H, Omoto K, Toki D, Iida S, Setoguchi S, Tokumoto T, Horita S, Nakayama H, Yamaguchi Y, Tanabe K: Analysis of renal transplant protocol biopsies in $\mathrm{ABO}$ incompatible kidney transplantation. Am J Transplant 2008;8:86-94.

34 Haas M, Segev DL, Racusen LC, Bagnasco SM, Locke JE, Warren DS, Simpkins CE, Lepley D, King KE, Kraus ES, Montgomery RA: C4d deposition without rejection correlates with reduced early scarring in $\mathrm{ABO}$-incompatible renal allografts. J Am Soc Nephrol 2009;20:197-204.

35 Rose ML, Smith J, Dureau G, Keogh A, Kobashigowa J: Mycophenolate mofetil decreases antibody production after cardiac transplantation. J Heart Lung Transplant 2002;21: 282-285.

36 Rentenaar RJ, van Diepen FN, Meijer RT, Surachno S, Wilmink JM, Schellekens PT, Pals ST, van Lier RA, ten Berge IJ: Immune responsiveness in renal transplant recipients: mycophenolic acid severely depresses humoral immunity in vivo. Kidney Int 2002;62: 319-328.

- 37 Genberg H, Kumlien G, Wennberg L, Tyden G: Isoagglutinin adsorption in ABO-incompatible transplantation. Trans Apher Sci 2010;43:231-235. 